\title{
A Meshless Method for the Numerical Solution of a Two-Dimension IHCP
}

\author{
F. Parzlivand and A. M. Shahrezaee \\ Department of Mathematics, Alzahra University, Vanak, Tehran 19834, Iran \\ Correspondence should be addressed to A. M. Shahrezaee; ashahrezaee@alzahra.ac.ir
}

Received 25 February 2014; Accepted 10 July 2014; Published 3 August 2014

Academic Editor: Ting-Zhu Huang

Copyright (C) 2014 F. Parzlivand and A. M. Shahrezaee. This is an open access article distributed under the Creative Commons Attribution License, which permits unrestricted use, distribution, and reproduction in any medium, provided the original work is properly cited.

\begin{abstract}
This paper uses the collocation method and radial basis functions (RBFs) to analyze the solution of a two-dimension inverse heat conduction problem (IHCP). The accuracy of the method is tested in terms of Error and RMS errors. Also, the stability of the technique is investigated by perturbing the additional specification data by increasing the amounts of random noise. The results of numerical experiments are compared with the analytical solution in illustrative examples to confirm the accuracy and efficiency of the presented scheme.
\end{abstract}

\section{Introduction}

In many industrial applications one wishes to determine the temperature on the surface of a body, where the surface itself is inaccessible for measurements. In this case, it is necessary to determine the surface temperature from a measured temperature history at a fixed location inside the body. This is called an IHCP and has been an interesting subject recently [1]. Inverse problems have practical implications in thermal transport systems which involve conduction, convection, and radiation. In thermal radiation [2], for example, identifying the distribution of the radiation source has been stimulated by a wide range of applications, including thermal control in space technology, combustion, high-temperature forming and coating technology, solar energy utilization, high-temperature engines, and furnace technology [3]. The importance of inverse heat conduction problems and appropriate solution algorithms are established in numerous works and the books (see, e.g., $[1,4-7]$ and the references therein). Several techniques have been proposed for solving a one-dimensional IHCP [8-14]. Among the methods proposed for higher dimensional IHCP, boundary element [15], finite difference [16], and finite element [17] have been widely adopted for problems in two-dimension. Besides, the sequential function specification method $[1,15]$ and differential method [18] have also been used in solving the IHCP. There is, however, still a need on numerical scheme for two-dimensional IHCP. The traditional meshdependent finite difference and finite element methods are so far the principal numerical tool of choice for the modeling and simulation of the IHCP. A major disadvantage of these methods, however, is their mesh-dependent characteristics which normally requires enormous computational effort and induces numerical instability when large number of grids or elements are required.

In this paper, a two-dimensional IHCP is solved by RBFs as a truly meshless/meshfree method. A meshfree method does not require a mesh to discretize the domain or boundary of the problem under consideration and the approximate solution is constructed entirely based on a set of scattered nodes. It is considered as the main advantage of these methods over the mesh-dependent techniques.

In the recent rapid development of meshless computational schemes, the RBF has been successfully developed as an efficient meshless scheme for solving various kinds of partial differential equations (PDEs) and ordinary differential equations (ODEs). For example, see [19-25]. Also some applications of this approach in solving inverse problems can be found in $[20,21,26,27]$. 


\section{Problem Formulation}

In this section, we consider the following two-dimension IHCP, in the dimensionless form:

$$
\begin{gathered}
U_{t}=U_{x x}+U_{y y} ; \quad 0<x<1,0<y<1,0<t<t_{\text {fin }}, \\
U(x, y, 0)=f(x, y) ; \quad 0 \leq x \leq 1,0 \leq y \leq 1, \\
U(0, y, t)=g_{0}(y, t) ; \quad 0 \leq y \leq 1,0 \leq t \leq t_{\text {fin }}, \\
U(1, y, t)=g_{1}(y, t) ; \quad 0 \leq y \leq 1,0 \leq t \leq t_{\text {fin }}, \\
U(x, 0, t)=h_{0}(x, t) ; \quad 0 \leq x \leq 1,0 \leq t \leq t_{\text {fin }}, \\
U(x, 1, t)=h_{1}(x, t) ; \quad 0 \leq x \leq 1,0 \leq t \leq t_{\text {fin }},
\end{gathered}
$$

and the overspecified condition:

$$
U\left(x_{1}, y, t\right)=E(y, t) ; \quad 0 \leq y \leq 1,0 \leq t \leq t_{\text {fin }},
$$

where $x_{1} \in(0,1)$ is known, $t_{\text {fin }}$ represent the final time of interest for the time evolution of the problem, and $f, g_{1}, h_{0}$, $h_{1}$, and $E$ are known functions in their domain satisfying the compatibility conditions while $U(x, y, t)$ and $g_{0}(y, t)$ remain to be determined from some interior temperature measurements.

The existence and uniqueness of the solution of this problem are discussed in [28]. The problem (1)-(2) may be divided into two separate problems.

The first problem is

$$
\begin{gathered}
U_{t}=U_{x x}+U_{y y} ; \quad x_{1}<x<1,0<y<1,0<t<t_{\text {fin }} \\
U(x, y, 0)=f(x, y) ; \quad x_{1} \leq x \leq 1,0 \leq y \leq 1, \\
U\left(x_{1}, y, t\right)=E(y, t) ; \quad 0 \leq y \leq 1,0 \leq t \leq t_{\text {fin }} \\
U(1, y, t)=g_{1}(y, t) ; \quad 0 \leq y \leq 1,0 \leq t \leq t_{\text {fin }} \\
U(x, 0, t)=h_{0}(x, t) ; \quad x_{1} \leq x \leq 1,0 \leq t \leq t_{\text {fin }} \\
U(x, 1, t)=h_{1}(x, t) ; \quad x_{1} \leq x \leq 1,0 \leq t \leq t_{\text {fin }} .
\end{gathered}
$$

This problem may be analyzed as a direct problem, for the portion of the body from $x=x_{1}$ to $x=1$ with known boundary conditions. There is a unique stable solution to the direct problem (3)-(8) and may be found in [29]. The second problem is the following IHCP:

$$
\begin{gathered}
U_{t}=U_{x x}+U_{y y} ; \quad 0<x<x_{1}, \quad 0<y<1,0<t<t_{\text {fin }} \\
U(x, y, 0)=f(x, y) ; \quad 0 \leq x \leq x_{1}, 0 \leq y \leq 1 \\
U(0, y, t)=g_{0}(y, t) ; \quad 0 \leq y \leq 1,0 \leq t \leq t_{\text {fin }} \\
U\left(x_{1}, y, t\right)=E(y, t) ; \quad 0 \leq y \leq 1,0 \leq t \leq t_{\text {fin }} \\
U(x, 0, t)=h_{0}(x, t) ; \quad 0 \leq x \leq x_{1}, 0 \leq t \leq t_{\text {fin }} \\
U(x, 1, t)=h_{1}(x, t) ; \quad 0 \leq x \leq x_{1}, \quad 0 \leq t \leq t_{\text {fin }}
\end{gathered}
$$

Therefore, for solving the inverse problem (1)-(2), we will investigate the direct problem (3)-(8) and IHCP (9)-(14). In
TABLE 1: Some well-known functions that generate RBFs.

\begin{tabular}{lc}
\hline Name of radial basis function & Definition \\
\hline Multiquadric (MQ) & $\varphi(r)=\sqrt{c^{2}+r^{2}}$ \\
Inverse quadratic (IQ) & $\varphi(r)=\frac{1}{\left(c^{2}+r^{2}\right)}$ \\
Inverse multiquadric (IMQ) & $\varphi(r)=\frac{1}{\sqrt{c^{2}+r^{2}}}$ \\
Gaussian (GA) & $\varphi(r)=\exp \left(-c^{2} r^{2}\right)$ \\
Thin plate splines (TPS) & $\varphi(r)=r^{2} \log (r)$ \\
\hline
\end{tabular}

the next section, the above problems will be considered; the heat in the body and heat at the boundary $x=0$ will be obtained by solving these problems numerically.

\section{Radial Basis Functions}

Radial basis functions are very efficient instruments for interpolating a scattered set of points. The use of the radial basis function for solving partial differential equations has some advantages over mesh-dependent methods, such as finite difference methods, finite element methods, spectral methods, finite volume methods, and boundary element methods. The use of radial basis functions as a meshless method for numerical solution of partial differential equations is based on the collocation method. Because of the collocation technique, this method does not need to evaluate any integral.

3.1. Definition of the Space Radial Basis Functions. Let $\mathbb{R}^{+}=$ $\{x \in \mathbb{R}, x \geq 0\},\|\cdot\|_{2}$ denote the Euclidean norm and let $\varphi$ : $\mathbb{R}^{+} \rightarrow \mathbb{R}$ be a continuous function with $\varphi(0) \geq 0$. A radial basis function on $\mathbb{R}^{d} ; d=1,2,3$ is a function of the form

$$
\phi_{s}(\mathbf{x})=\varphi\left(\left\|\mathbf{x}-\mathbf{x}_{s}\right\|_{2}\right)
$$

which depended only on the distance between $\mathbf{x} \in \mathbb{R}^{d}$ and a fixed point $\mathbf{x}_{s} \in \mathbb{R}^{d}$ [30]. So that the radial basis function $\phi_{s}$ is radially symmetric about the center $\mathbf{x}_{s}$. Some best-known RBFs are listed in Table 1, where $r=\left\|\mathbf{x}-\mathbf{x}_{s}\right\|_{2}$ and $c$ is a free positive parameter, often referred to as the shape parameter, to be specified by the user. Despite many research works, which have been done to find algorithms for selecting the optimum values of $c$ [31-33], the optimal choice of shape parameter is an open problem, which is still under intensive investigation.

The standard radial basis functions are categorized into two major classes $[34,35]$.

Class 1. Infinitely smooth RBFs.

These basis functions are infinitely differentiable and involve a parameter $c$ (such as multiquadric (MQ), inverse multiquadric (IMQ), and Gaussian) which needs to be selected so that the required accuracy of the solution is attained.

Class 2. Infinitely smooth (except at centers) RBFs. 
These basis functions are not infinitely differentiable. These basis functions are shape parameter free and have comparatively less accuracy than the basis functions discussed in Class 1. Examples are thin plate splines.

\section{Numerical Procedures}

Now we use the RBFs for discretization of both time and space variables. Let $\left\{\left(x_{i}, y_{j}, t_{k}\right), 0 \leq x_{i} \leq 1,0 \leq y_{j} \leq 1,0 \leq t_{k} \leq t_{\text {fin }}\right.$, $i=1, \ldots, N, j=1, \ldots, M, k=1, \ldots, S\}$ be a set of scattered nodes. Then, the function $U(x, y, t)$ of two spatial dimensions to be interpolated can be represented by RBFs as

$$
\widetilde{U}(x, y, t) \simeq \sum_{i=1}^{N} \sum_{j=1}^{M} \sum_{k=1}^{S} \lambda_{i, j}^{k} \phi_{i, j}^{k}(x, y, t)
$$

where $\phi_{i, j}^{k}(x, y, t)=\varphi\left(\left\|(x, y, t)-\left(x_{i}, y_{j}, t_{k}\right)\right\|_{2}\right)$ for a radial function $\varphi$ and $\lambda_{i, j}^{k} ; i=1, \ldots, N, j=1, \ldots, M$, and $k=$ $1, \ldots, S$ are unknown constants that must be found.

4.1. The Application of RBFs in IHCP (9)-(14). For this problem, let $x_{i}=x_{1}((i-1) /(N-1)), i=1,2, \ldots, N ; y_{j}=$ $((j-1) /(M-1)), j=1,2, \ldots, M$; and $t_{k}=((k-1) /(S-1))$, $k=1,2, \ldots, S$. Now (9)-(14) are approximated using (16). The collocation technique is used for finding unknown constants $\lambda_{i, j}^{k}$.

We collocate $(9)$ in $(N-1) \times(M-2) \times(S-1)$ points $\Omega_{1}=$ $\left\{\left(x_{n}, y_{m}, t_{l}\right) ; n=1, \ldots, N-1, m=2, \ldots, M-1, l=2, \ldots, S\right\}$; we have

$$
\begin{array}{r}
\sum_{i=1}^{N} \sum_{j=1}^{M} \sum_{k=1}^{S} \lambda_{i, j}^{k}\left\{\frac{\partial}{\partial t} \phi_{i, j}^{k}\left(x_{n}, y_{m}, t_{l}\right)-\frac{\partial^{2}}{\partial x^{2}} \phi_{i, j}^{k}\left(x_{n}, y_{m}, t_{l}\right)\right. \\
\left.-\frac{\partial^{2}}{\partial y^{2}} \phi_{i, j}^{k}\left(x_{n}, y_{m}, t_{l}\right)\right\}=0 ; \\
\left(x_{n}, y_{m}, t_{l}\right) \in \Omega_{1} .
\end{array}
$$

Now, collocation (10) in $N \times M$ points $\Omega_{2}=\left\{\left(x_{n}, y_{m}, t_{l}\right) ; n=\right.$ $1, \ldots, N, m=1, \ldots, M, l=1\}$ yields

$$
\begin{array}{r}
\sum_{i=1}^{N} \sum_{j=1}^{M} \sum_{k=1}^{S} \lambda_{i, j}^{k} \phi_{i, j}^{k}\left(x_{n}, y_{m}, t_{l}\right)-f\left(x_{n}, y_{n}\right)=0 \\
\left(x_{n}, y_{m}, t_{l}\right) \in \Omega_{2} .
\end{array}
$$

By collocation (12) in $M \times(S-1)$ points $\Omega_{3}=\left\{\left(x_{n}, y_{m}, t_{l}\right) ; n=\right.$ $N, m=1, \ldots, M, l=2, \ldots, S\}$, we obtain

$$
\begin{array}{r}
\sum_{i=1}^{N} \sum_{j=1}^{M} \sum_{k=1}^{S} \lambda_{i, j}^{k} \phi_{i, j}^{k}\left(x_{n}, y_{m}, t_{l}\right)-E\left(y_{m}, t_{l}\right)=0 ; \\
\left(x_{n}, y_{m}, t_{l}\right) \in \Omega_{3} .
\end{array}
$$

By collocating (13) in $(N-1) \times(S-1)$ points $\Omega_{4}=$ $\left\{\left(x_{n}, y_{m}, t_{l}\right) ; n=1, \ldots, N-1, m=1, l=2, \ldots, S\right\}$, we can write

$$
\begin{aligned}
\sum_{i=1}^{N} \sum_{j=1}^{M} \sum_{k=1}^{S} \lambda_{i, j}^{k} \phi_{i, j}^{k}\left(x_{n}, y_{m}, t_{l}\right)-h_{0}\left(x_{n}, t_{l}\right) & =0 ; \\
\left(x_{n}, y_{m}, t_{l}\right) & \in \Omega_{4},
\end{aligned}
$$

and by collocation (14) in $(N-1) \times(S-1)$ points $\Omega_{5}=$ $\left\{\left(x_{n}, y_{m}, t_{l}\right) ; n=1, \ldots, N-1, m=M, l=2, \ldots, S\right\}$, we have

$$
\begin{aligned}
\sum_{i=1}^{N} \sum_{j=1}^{M} \sum_{k=1}^{S} \lambda_{i, j}^{k} \phi_{i, j}^{k}\left(x_{n}, y_{m}, t_{l}\right)-h_{1}\left(x_{n}, t_{l}\right) & =0 \\
\left(x_{n}, y_{m}, t_{l}\right) & \in \Omega_{5} .
\end{aligned}
$$

Equations (17)-(21) give a $N \times M \times S$ system of linear algebraic equations with the $N \times M \times S$ unknown coefficients $\lambda_{i, j}^{k}$. Solving this linear system, the approximate temperature distribution of the problem (9)-(14) is obtained. Knowing the temperature distribution, we can easily determine the function describing the boundary condition:

$$
g_{0}(y, t) \simeq \widetilde{U}(0, y, t) .
$$

4.2. The Application of RBFs in Direct Problem (3)-(8). Now, let $x_{i}=x_{1}+\left(1-x_{1}\right)((i-1) /(N-1)) ; i=1,2, \ldots, N, y_{j}=$ $(j-1) /(M-1) ; j=1,2, \ldots, M$, and $t_{k}=(k-1) /(S-1) ; k=$ $1,2, \ldots, S$. The unknown function $U(x, y, t)$ in $(3)-(8)$ can be approximated by using (16). The collocation technique is used for finding unknown coefficients $\lambda_{i, j}^{k}$.

We collocate $(3)$ in $(N-1) \times(M-2) \times(S-1)$ points $\Gamma_{1}=$ $\left\{\left(x_{n}, y_{m}, t_{l}\right) ; n=1, \ldots, N-1, m=2, \ldots, M-1, l=2, \ldots, S\right\}$; we get

$$
\begin{array}{r}
\sum_{i=1}^{N} \sum_{j=1}^{M} \sum_{k=1}^{S} \lambda_{i, j}^{k}\left\{\frac{\partial}{\partial t} \phi_{i, j}^{k}\left(x_{n}, y_{m}, t_{l}\right)-\frac{\partial^{2}}{\partial x^{2}} \phi_{i, j}^{k}\left(x_{n}, y_{m}, t_{l}\right)\right. \\
\left.-\frac{\partial^{2}}{\partial y^{2}} \phi_{i, j}^{k}\left(x_{n}, y_{m}, t_{l}\right)\right\}=0, \\
\left(x_{n}, y_{m}, t_{l}\right) \in \Gamma_{1} .
\end{array}
$$

Now, collocation (4) in $N \times M$ points $\Gamma_{2}=\left\{\left(x_{n}, y_{m}, t_{l}\right) ; n=\right.$ $1, \ldots, N, m=1, \ldots, M, l=1\}$, yields

$$
\begin{aligned}
\sum_{i=1}^{N} \sum_{j=1}^{M} \sum_{k=1}^{S} \lambda_{i, j}^{k} \phi_{i, j}^{k}\left(x_{n}, y_{m}, t_{l}\right)-f\left(x_{n}, y_{m}\right) & =0 ; \\
\left(x_{n}, y_{m}, t_{l}\right) & \in \Gamma_{2} .
\end{aligned}
$$

By collocation (5) in $M \times(S-1)$ points $\Gamma_{3}=\left\{\left(x_{n}, y_{m}, t_{l}\right) ; n=\right.$ $1, m=1, \ldots, M, l=2, \ldots, S\}$, we obtain

$$
\begin{aligned}
\sum_{i=1}^{N} \sum_{j=1}^{M} \sum_{k=1}^{S} \lambda_{i, j}^{k} \phi_{i, j}^{k}\left(x_{n}, y_{m}, t_{l}\right)-E\left(y_{m}, t_{l}\right) & =0 ; \\
\left(x_{n}, y_{m}, t_{l}\right) & \in \Gamma_{3} .
\end{aligned}
$$


TABLE 2: The comparison between the exact, Tikhonov, SVD, and GA-RBFs solution for $g_{0}(y, 0.04)$ with noiseless data for Example 1 .

\begin{tabular}{lccccccc}
\hline$y$ & Exact & Tikhonov 0 & Tikhonov 1 & Tikhonov 2 & SVD & $\begin{array}{c}\text { GA-RBFs } \\
(N=M=S=5)\end{array} \quad \begin{array}{c}\text { GA-RBFs } \\
(N=11, M=S=8)\end{array}$ \\
\hline 0.1 & 0.955989 & 0.977515 & 0.953963 & 0.953359 & 0.977522 & 0.956061 & 0.955988 \\
0.2 & 0.941638 & 0.961234 & 0.937549 & 0.937005 & 0.961242 & 0.941742 & 0.941636 \\
0.3 & 0.917877 & 0.936400 & 0.912705 & 0.912258 & 0.936409 & 0.918004 & 0.917875 \\
0.4 & 0.884946 & 0.903014 & 0.879430 & 0.879118 & 0.903023 & 0.885090 & 0.884943 \\
0.5 & 0.843172 & 0.861074 & 0.837723 & 0.837584 & 0.861083 & 0.843326 & 0.843169 \\
0.6 & 0.792974 & 0.810581 & 0.787585 & 0.787658 & 0.810590 & 0.793124 & 0.792971 \\
0.7 & 0.734852 & 0.751535 & 0.729016 & 0.729339 & 0.751544 & 0.734981 & 0.734850 \\
0.8 & 0.669388 & 0.683935 & 0.662016 & 0.662626 & 0.683945 & 0.669479 & 0.669387 \\
0.9 & 0.597236 & 0.607783 & 0.586884 & 0.587521 & 0.607793 & 0.597279 & 0.597935 \\
\hline
\end{tabular}

TABLE 3: The comparison between the exact, Tikhonov, SVD, and GA-RBFs solution for $g_{0}(y, 0.04)$ with noisy data $(\delta=0.01)$ for Example 1 .

\begin{tabular}{lccccccc}
\hline$y$ & Exact & Tikhonov 0 & Tikhonov 1 & Tikhonov 2 & SVD & $\begin{array}{c}\text { GA-RBFs } \\
(N=M=S=5)\end{array} \quad \begin{array}{c}\text { GA-RBFs } \\
(N=11, M=S=8)\end{array}$ \\
\hline 0.1 & 0.955989 & 0.989553 & 0.959390 & 0.958599 & 0.989560 & 0.956861 & 0.955980 \\
0.2 & 0.941638 & 0.971284 & 0.940893 & 0.940179 & 0.971291 & 0.941096 & 0.941640 \\
0.3 & 0.917877 & 0.945030 & 0.914534 & 0.913948 & 0.945037 & 0.919012 & 0.917873 \\
0.4 & 0.884946 & 0.910791 & 0.880313 & 0.879905 & 0.910798 & 0.880111 & 0.884940 \\
0.5 & 0.843172 & 0.868567 & 0.838231 & 0.838050 & 0.868575 & 0.843935 & 0.843171 \\
0.6 & 0.792974 & 0.818358 & 0.788287 & 0.788384 & 0.818366 & 0.793001 & 0.792979 \\
0.7 & 0.734852 & 0.760165 & 0.730482 & 0.730907 & 0.760172 & 0.735098 & 0.734855 \\
0.8 & 0.669388 & 0.693986 & 0.664816 & 0.665618 & 0.693994 & 0.669153 & 0.597291 \\
0.9 & 0.597236 & 0.619823 & 0.591288 & 0.592518 & 0.619830 & & 0.597938 \\
\hline
\end{tabular}

By collocation (6) in $M \times(S-1)$ points $\Gamma_{4}=\left\{\left(x_{n}, y_{m}, t_{l}\right) ; n=\right.$ $N, m=1, \ldots, M, l=2, \ldots, S\}$, we can write

$$
\begin{aligned}
\sum_{i=1}^{N} \sum_{j=1}^{M} \sum_{k=1}^{S} \lambda_{i, j}^{k} \phi_{i, j}^{k}\left(x_{n}, y_{m}, t_{l}\right)-g_{1}\left(x_{n}, t_{l}\right) & =0 ; \\
\left(x_{n}, y_{m}, t_{l}\right) & \in \Gamma_{4} .
\end{aligned}
$$

By collocation (7) in $(N-2) \times(S-1)$ points $\Gamma_{5}=$ $\left\{\left(x_{n}, y_{m}, t_{l}\right) ; n=2, \ldots, N-1, m=1, l=2, \ldots, S\right\}$, we have

$$
\begin{aligned}
\sum_{i=1}^{N} \sum_{j=1}^{M} \sum_{k=1}^{S} \lambda_{i, j}^{k} \phi_{i, j}^{k}\left(x_{n}, y_{m}, t_{l}\right)-h_{0}\left(x_{n}, t_{l}\right) & =0 ; \\
\left(x_{n}, y_{m}, t_{l}\right) & \in \Gamma_{5},
\end{aligned}
$$

and by collocation $(8)$ in $(N-2) \times(S-1)$ points $\Gamma_{6}=$ $\left.\left\{x_{n}, y_{m}, t_{l}\right) ; n=2, \ldots, N-1, m=M, l=2, \ldots, S\right\}$, we have

$$
\begin{array}{r}
\sum_{i=1}^{N} \sum_{j=1}^{M} \sum_{k=1}^{S} \lambda_{i, j}^{k} \phi_{i, j}^{k}\left(x_{n}, y_{m}, t_{l}\right)-h_{1}\left(x_{n}, t_{l}\right)=0 ; \\
\left(x_{n}, y_{m}, t_{l}\right) \in \Gamma_{6} .
\end{array}
$$

Equations (23) -(28) give a $N \times M \times S$ system of linear algebraic equations with the $N \times M \times S$ unknown coefficients $\lambda_{i, j}^{k}$. Solving this linear system, the approximate solution of the problem (3)-(8) is obtained.

\section{Convergence Analysis and Error Bound}

This section covers the error analysis of the proposed method. Also the sufficient conditions are presented to guarantee the convergence of RBFs, when applied to solve the differential equations.

5.1. Approximation Error. Here, we are concerned with the error of the approximation of a given three-variate function by its expansion in terms of radial basis functions.

Suppose that $X=L^{2}(\Omega)$, where $\Omega=[0,1] \times[0,1] \times\left[0, t_{\text {fin }}\right]$ (also note that the solution $u \in C^{4,4,2}(\Omega) \subset L^{2}(\Omega)$ ); the inner product in this space is defined by

$$
\begin{aligned}
& \left\langle f_{1}(x, y, t), f_{2}(x, y, t)\right\rangle \\
& \quad=\int_{0}^{t_{\text {fin }}} \iint_{0}^{1} f_{1}(x, y, t) f_{2}(x, y, t) d x d y d t,
\end{aligned}
$$

and the norm is as follows:

$$
\begin{aligned}
\| f & (x, y, t) \| \\
& =\langle f(x, y, t), f(x, y, t)\rangle^{1 / 2} \\
& =\left(\int_{0}^{t_{\text {fin }}} \iint_{0}^{1}|f(x, y, t)|^{2} d x d y d t\right)^{1 / 2} .
\end{aligned}
$$


TABLE 4: Error and RMS errors for $U(x, y, t)$ and $g_{0}(y, t)$ on the intervals $x \in[0,0.1], y \in[0,1]$, and $t \in[0,1]$ for Example 1 .

\begin{tabular}{|c|c|c|c|c|c|}
\hline Method & Error & $N=M=S=3$ & $N=6, M=S=4$ & $N=5, M=4, S=6$ & $N=7, M=5, S=6$ \\
\hline \multirow{4}{*}{$\begin{array}{l}\text { GA-RBFs } \\
(c=0.1)\end{array}$} & $\operatorname{RMS}(U)$ & $1.800 E-3$ & $3.782 E-4$ & $2.253 E-4$ & $3.749 E-5$ \\
\hline & $\operatorname{Error}(U)$ & $2.894 E-3$ & $6.169 E-4$ & $3.748 E-4$ & $6.209 E-5$ \\
\hline & $\operatorname{RMS}\left(g_{0}\right)$ & $2.795 E-3$ & $6.242 E-4$ & $3.672 E-4$ & $6.271 E-5$ \\
\hline & Error $\left(g_{0}\right)$ & $4.763 E-3$ & $1.078 E-3$ & $6.470 E-4$ & $1.099 E-4$ \\
\hline \multirow{4}{*}{$\begin{array}{l}\text { IMQ-RBFs } \\
(c=50)\end{array}$} & $\operatorname{RMS}(U)$ & $1.506 E-3$ & $9.596 E-4$ & $7.356 E-4$ & $8.187 E-5$ \\
\hline & $\operatorname{Error}(U)$ & $2.421 E-3$ & $1.692 E-3$ & $1.223 E-3$ & $1.356 E-4$ \\
\hline & $\operatorname{RMS}\left(g_{0}\right)$ & $2.334 E-3$ & $2.020 E-3$ & $1.201 E-3$ & $1.370 E-4$ \\
\hline & $\operatorname{Error}\left(g_{0}\right)$ & $3.977 E-3$ & $3.111 E-3$ & $2.117 E-3$ & $2.402 E-4$ \\
\hline \multirow{4}{*}{$\begin{array}{l}\text { MQ-RBFs } \\
(c=50)\end{array}$} & $\operatorname{RMS}(U)$ & $1.378 E-3$ & $9.301 E-3$ & $1.135 E-3$ & $1.638 E-4$ \\
\hline & $\operatorname{Error}(U)$ & $2.216 E-3$ & $2.093 E-3$ & $1.888 E-3$ & $2.693 E-4$ \\
\hline & $\operatorname{RMS}\left(g_{0}\right)$ & $2.135 E-3$ & $2.101 E-3$ & $1.853 E-3$ & $2.689 E-4$ \\
\hline & Error $\left(g_{0}\right)$ & $3.639 E-3$ & $3.491 E-3$ & $3.266 E-3$ & $4.682 E-4$ \\
\hline \multirow{4}{*}{$\begin{array}{l}\text { IQ-RBFs } \\
(c=50)\end{array}$} & $\operatorname{RMS}(U)$ & $1.563 E-3$ & $9.301 E-3$ & $1.172 E-3$ & $6.588 E-5$ \\
\hline & $\operatorname{Error}(U)$ & $2.514 E-3$ & $2.093 E-3$ & $1.951 E-3$ & $1.091 E-4$ \\
\hline & $\operatorname{RMS}\left(g_{0}\right)$ & $2.423 E-3$ & $2.101 E-3$ & $1.913 E-3$ & $1.104 E-4$ \\
\hline & $\operatorname{Error}\left(g_{0}\right)$ & $4.129 E-3$ & $3.491 E-3$ & $3.371 E-3$ & $1.936 E-4$ \\
\hline
\end{tabular}

TABLE 5: Error and RMS errors for $U(x, y, t)$ on the intervals $x \in[0.1,1], y \in[0,1]$, and $t \in[0,1]$ for Example 1 .

\begin{tabular}{|c|c|c|c|c|c|}
\hline Method & Error & $N=M=S=3$ & $N=6, M=S=4$ & $N=5, M=4, S=6$ & $N=7, M=5, S=6$ \\
\hline \multirow{2}{*}{$\begin{array}{l}\text { GA-RBFs } \\
(c=0.1)\end{array}$} & $\operatorname{RMS}(U)$ & $7.237 E-4$ & $3.782 E-4$ & $1.038 E-4$ & $3.361 E-6$ \\
\hline & $\operatorname{Error}(U)$ & $7.611 E-4$ & $6.169 E-4$ & $1.132 E-4$ & $3.625 E-6$ \\
\hline \multirow{2}{*}{$\begin{array}{l}\text { IMQ-RBFs } \\
(c=50)\end{array}$} & $\operatorname{RMS}(U)$ & $1.342 E-4$ & $6.216 E-4$ & $5.188 E-4$ & $2.424 E-6$ \\
\hline & $\operatorname{Error}(U)$ & $1.412 E-4$ & $6.450 E-4$ & $5.657 E-4$ & $2.634 E-6$ \\
\hline \multirow{2}{*}{$\begin{array}{l}\text { MQ-RBFs } \\
(c=50)\end{array}$} & $\operatorname{RMS}(U)$ & $6.635 E-3$ & $4.011 E-3$ & $1.187 E-3$ & $9.449 E-6$ \\
\hline & $\operatorname{Error}(U)$ & $6.978 E-3$ & $4.721 E-3$ & $2.043 E-3$ & $1.026 E-5$ \\
\hline \multirow{2}{*}{$\begin{array}{l}\text { IQ-RBFs } \\
(c=50)\end{array}$} & $\operatorname{RMS}(U)$ & $1.181 E-3$ & $8.112 E-4$ & $4.305 E-4$ & $2.424 E-6$ \\
\hline & $\operatorname{Error}(U)$ & $1.904 E-4$ & $8.430 E-4$ & $4.694 E-4$ & $2.634 E-6$ \\
\hline
\end{tabular}

Let $\left\{\phi_{1}(x, y, t), \phi_{2}(x, y, t), \ldots, \phi_{N}(x, y, t)\right\} \subset X$ be the set of radial basis functions and

$$
Y=\operatorname{span}\left\{\phi_{1}(x, y, t), \phi_{2}(x, y, t), \ldots, \phi_{N}(x, y, t)\right\},
$$

and let $f$ be an arbitrary element in $X$. Since $Y$ is a finite dimensional vector space, $f$ has the unique best approximation out of $Y$ such as $f_{0} \in Y$ [36], such that

$$
\forall y \in Y, \quad\left\|f-f_{0}\right\| \leq\|f-y\| .
$$

Moreover, since $f_{0} \in Y$, there exist unique coefficients $\gamma_{1}, \gamma_{2}, \ldots, \gamma_{N}$ such that

$$
f(x, y, t) \simeq f_{0}(x, y, t)=\sum_{i=1}^{N} \gamma_{i} \phi_{i}(x, y, t) .
$$

Theorem 1. Let $(X,\|\cdot\|)$ be a Hilbert space and let $Y$ be a closed subspace of $X$ such that $\operatorname{dim} Y<\infty$ and $\left\{y_{1}, y_{2}, \ldots, y_{n}\right\}$ is any basis for $Y$. Let $f$ be an arbitrary element in $X$ and let $f_{0}$ be the unique best approximation to $f$ out of $Y$. Then [36]

$$
\left\|f-f_{0}\right\|=\frac{G\left(f, y_{1}, y_{2}, \ldots, y_{n}\right)}{G\left(y_{1}, y_{2}, \ldots, y_{n}\right)},
$$

where

$$
\begin{aligned}
G & \left(y_{1}, y_{2}, \ldots, y_{n}\right) \\
& =\left|\begin{array}{cccc}
\left\langle y_{1}, y_{1}\right\rangle & \left\langle y_{1}, y_{2}\right\rangle & \ldots & \left\langle y_{1}, y_{n}\right\rangle \\
\left\langle y_{2}, y_{1}\right\rangle & \left\langle y_{2}, y_{2}\right\rangle & \ldots & \left\langle y_{2}, y_{n}\right\rangle \\
\vdots & \vdots & \ddots & \vdots \\
\left\langle y_{n}, y_{1}\right\rangle & \left\langle y_{n}, y_{2}\right\rangle & \cdots & \left\langle y_{n}, y_{n}\right\rangle
\end{array}\right| .
\end{aligned}
$$

5.2. Convergence Analysis. We have the following theorem about the convergence of RBFs interpolation.

Theorem 2. Assume $\left\{x_{i}\right\}_{i=1}^{N}$ are $N$ nodes in $\Omega \subseteq \mathbb{R}^{d}$ which is convex; let

$$
h=\max _{x \in \Omega} \min _{1 \leq i \leq N}\left\|x-x_{i}\right\|_{2}
$$

when $\phi(\eta)<c(1+|\eta|)^{-(2 l+d)}$ for any $y(x)$ satisfies $\int(y(\eta))^{2} / \phi(\eta) d \eta<\infty$; we have

$$
\left\|y_{N}^{(\alpha)}-y^{(\alpha)}\right\|_{\infty} \leq c h^{1-\alpha},
$$


TABle 6: The comparison between the exact, Tikhonov, SVD, and GA-RBFs solution for $g_{0}(y, 0.04)$ with noiseless data for Example 2.

\begin{tabular}{lccccccc}
\hline$y$ & Exact & Tikhonov 0 & Tikhonov 1 & Tikhonov 2 & SVD & $\begin{array}{c}\text { GA-RBFs } \\
(N=M=S=5)\end{array} \quad \begin{array}{c}\text { GA-RBFs } \\
(N=11, M=S=8)\end{array}$ \\
\hline 0.1 & 0.095591 & 0.099151 & 0.091232 & 0.091254 & 0.099229 & 0.095594 & 0.095591 \\
0.2 & 0.190879 & 0.194718 & 0.188768 & 0.188793 & 0.194778 & 0.190958 & 0.190879 \\
0.3 & 0.283933 & 0.286510 & 0.282289 & 0.282316 & 0.286555 & 0.284056 & 0.283932 \\
0.4 & 0.374149 & 0.374528 & 0.371793 & 0.371824 & 0.374561 & 0.374295 & 0.374148 \\
0.5 & 0.460627 & 0.454773 & 0.457291 & 0.457315 & 0.458794 & 0.460774 & 0.460626 \\
0.6 & 0.542503 & 0.539243 & 0.538753 & 0.538791 & 0.539256 & 0.542638 & 0.542502 \\
0.7 & 0.618958 & 0.615939 & 0.616208 & 0.616251 & 0.615946 & 0.619078 & 0.618957 \\
0.8 & 0.689228 & 0.688861 & 0.689647 & 0.689695 & 0.688864 & 0.689332 & 0.752690 \\
0.9 & 0.752612 & 0.758009 & 0.759070 & 0.759124 & 0.758011 & & 0.752612 \\
\hline
\end{tabular}

TABLE 7: The comparison between the exact, Tikhonov, SVD, and GA-RBFs solution for $g_{0}(y, 0.04)$ with noisy data $(\delta=0.01)$ for Example 2 .

\begin{tabular}{|c|c|c|c|c|c|c|c|}
\hline$y$ & Exact & Tikhonov 0 & Tikhonov 1 & Tikhonov 2 & SVD & $\begin{array}{c}\text { GA-RBFs } \\
(N=M=S=5)\end{array}$ & $\begin{array}{c}\text { GA-RBFs } \\
(N=11, M=S=8)\end{array}$ \\
\hline 0.1 & 0.095591 & 0.101437 & 0.097458 & 0.097523 & 0.101531 & 0.095599 & 0.095591 \\
\hline 0.2 & 0.190879 & 0.198779 & 0.195645 & 0.195653 & 0.198852 & 0.190983 & 0.190879 \\
\hline 0.3 & 0.283933 & 0.291784 & 0.289378 & 0.289331 & 0.291838 & 0.284318 & 0.283938 \\
\hline 0.4 & 0.374149 & 0.380451 & 0.378655 & 0.378557 & 0.380490 & 0.374808 & 0.374150 \\
\hline 0.5 & 0.460627 & 0.464780 & 0.463477 & 0.463330 & 0.464807 & 0.460512 & 0.460621 \\
\hline 0.6 & 0.542503 & 0.544772 & 0.543844 & 0.543652 & 0.544789 & 0.542840 & 0.542509 \\
\hline 0.7 & 0.618958 & 0.620427 & 0.619756 & 0.619522 & 0.620437 & 0.619578 & 0.618957 \\
\hline 0.8 & 0.689228 & 0.691744 & 0.691212 & 0.690939 & 0.691750 & 0.689809 & 0.689221 \\
\hline 0.9 & 0.752612 & 0.758723 & 0.758213 & 0.757905 & 0.758728 & 0.752831 & 0.752612 \\
\hline
\end{tabular}

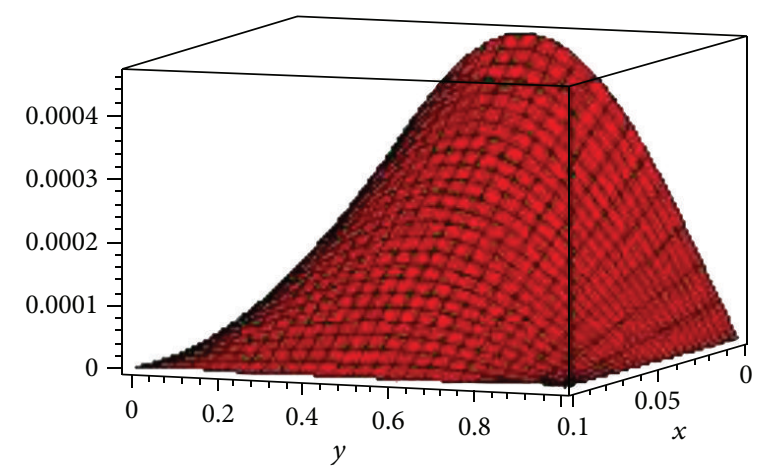

(a) $|U(x, y, 1)-\widetilde{U}(x, y, 1)|$ for $N=M=S=5$

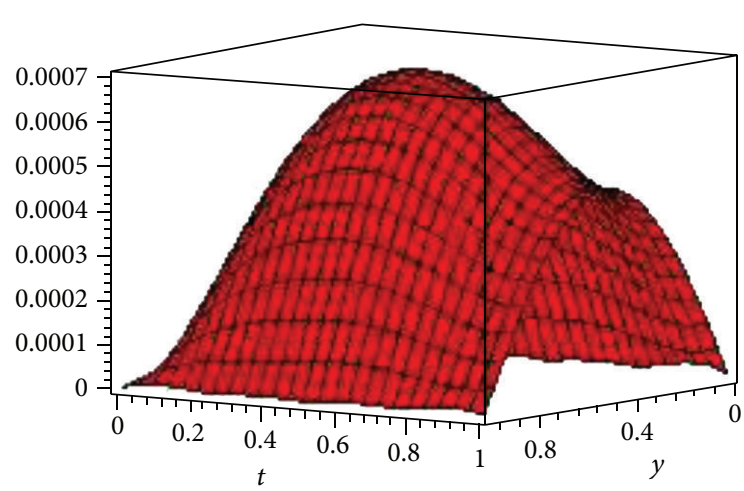

(c) $\left|g_{0}(y, t)-\widetilde{U}(0, y, t)\right|$ for $N=M=S=5$

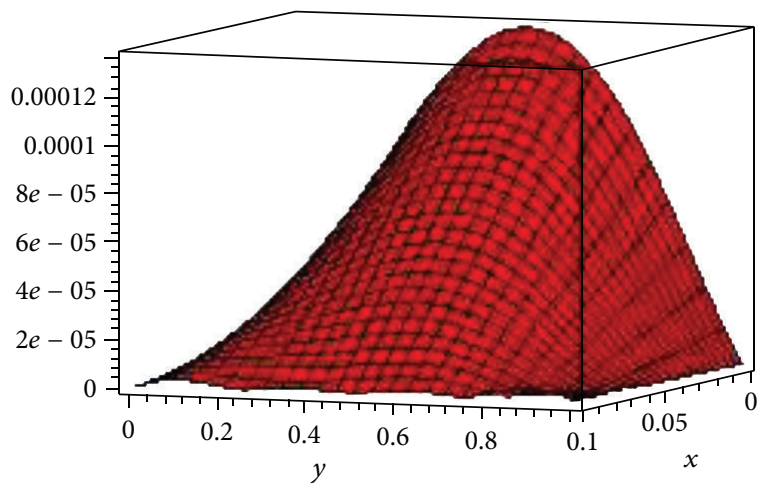

(b) $|U(x, y, 1)-\widetilde{U}(x, y, 1)|$ for $N=7, M=5, S=6$

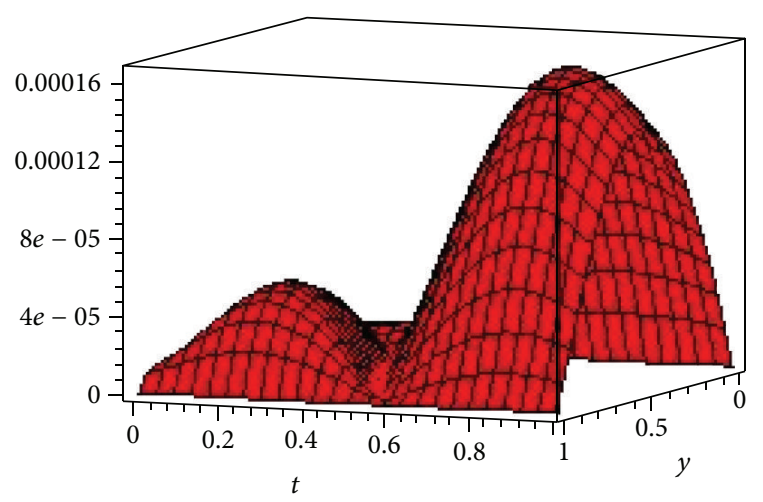

(d) $\left|g_{0}(y, t)-\widetilde{U}(0, y, t)\right|$ for $N=7, M=5, S=6$

FIGURE 1: Graph of absolute error for $U$ and $g_{0}$ by using GA-RBF with $c=0.1$ for Example 1 . 


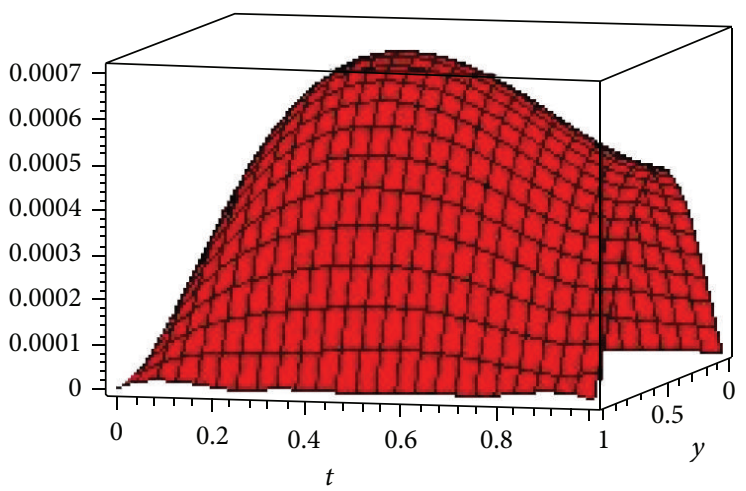

(a) $\left|g_{0}(y, t)-\widetilde{U}(0, y, t)\right|$ for GA-RBF with $c=0.1$

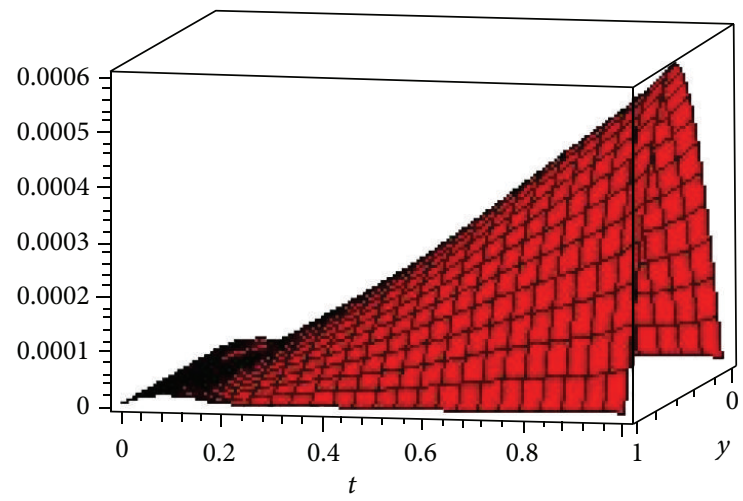

(c) $\left|g_{0}(y, t)-\widetilde{U}(0, y, t)\right|$ for MQ-RBF with $c=50$

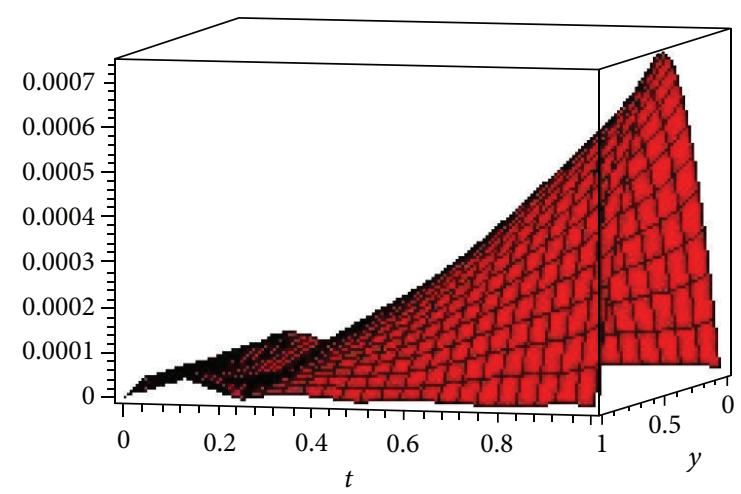

(b) $\left|g_{0}(y, t)-\widetilde{U}(0, y, t)\right|$ for IMQ-RBF with $c=50$

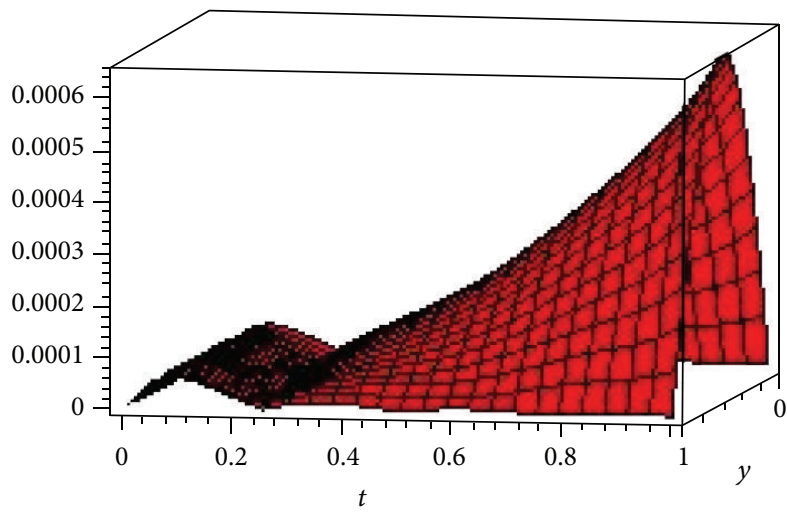

(d) $\left|g_{0}(y, t)-\widetilde{U}(0, y, t)\right|$ for IQ-RBF with $c=50$

FiguRE 2: Graph of absolute error for $g_{0}$ by using different RBFs for Example 2 with $N=5, M=6, S=5$.

where $\phi(x)$ is RBFs, the constant $c$ depends on the RBFs, $d$ is space dimension, and $l$ and $\alpha$ are nonnegative integer.

Proof. A complete proof is given by authors $[37,38]$.

It can be seen that not only RBFs themselves but also any of their order derivatives has a good convergence.

\section{Test Examples}

In order to illustrate the performance of the RBFs method in solving IHCPs and justify the accuracy and efficiency of the method presented in this paper, we consider the following examples. For two examples, the true solutions are available. We tested the accuracy and stability of the method presented in this paper by performing the mentioned method for different values of $N, M$, and $S$. To study the convergence behavior of the RBFs method, we applied the following laws.

(1) The error Error is described using

\section{$\operatorname{Error}(U)$}

$$
=\sqrt{\frac{\sum_{i=1}^{N} \sum_{j=1}^{M} \sum_{k=1}^{S}\left(U\left(x_{i}, y_{j}, t_{k}\right)-\widetilde{U}\left(x_{i}, y_{j}, t_{k}\right)\right)^{2}}{\sum_{i=1}^{N} \sum_{j=1}^{M} \sum_{k=1}^{S}\left(U\left(x_{i}, y_{j}, t_{k}\right)\right)^{2}}} .
$$

(2) The root mean square (RMS) is described using

\section{$\operatorname{RMS}(U)$}

$$
=\sqrt{\frac{1}{N M S} \sum_{i=1}^{N} \sum_{j=1}^{M} \sum_{k=1}^{S}\left|U\left(x_{i}, y_{j}, t_{k}\right)-\widetilde{U}\left(x_{i}, y_{j}, t_{k}\right)\right|^{2}},
$$

where $\left(x_{i}, y_{j}, t_{k}\right)$ are interpolate nodes, $U$ is the exact value, and $\widetilde{U}$ is the RBFs approximation.

In order to investigate the stability of the numerical method, the additional specification data has been perturbed as

$$
E(y, t)^{\delta}=E(y, t)[1+\delta \times \operatorname{rand}(x)]
$$

where $\delta$ is the relative (percentage) noise level and $\operatorname{rand}(x)$ is a random number between $(0,1)$.

6.1. Example 1. We solve the problem (1)-(2) with [28]: $x_{1}=$ $0.1, t_{\text {fin }}=1, f(x, y)=\sin (x)+\cos (y) g_{1}(y, t)=e^{-t}(\sin (1)+$ $\cos (y)), h_{0}(x, t)=e^{-t}(1+\sin (x)), h_{1}(y, t)=e^{-t}(\sin (x)+$ $\cos (1))$, and $E(y, t)=e^{-t}(\sin (0.1)+\cos (y))$ for which the exact solution is $u(x, y, t)=e^{-t}(\sin (x)+\cos (y))$ and $g_{0}(y, t)=$ $e^{-t} \cos (y)$. 
TABLE 8: Error and RMS errors for $U(x, y, t)$ and $g_{0}(y, t)$ on the intervals $x \in[0,0.1], y \in[0,1]$, and $t \in[0,1]$ for Example 2 .

\begin{tabular}{|c|c|c|c|c|c|}
\hline Method & Error & $N=M=S=3$ & $N=6, M=4, S=5$ & $N=M=S=5$ & $N=5, M=6, S=5$ \\
\hline \multirow{4}{*}{$\begin{array}{l}\text { GA-RBFs } \\
(c=0.1)\end{array}$} & $\operatorname{RMS}(U)$ & $1.801 E-3$ & $3.298 E-5$ & $2.040 E-5$ & $1.092 E-5$ \\
\hline & Error $(U)$ & $4.237 E-3$ & $8.186 E-5$ & $5.112 E-5$ & $4.281 E-5$ \\
\hline & $\operatorname{RMS}\left(g_{0}\right)$ & $2.797 E-3$ & $5.222 E-5$ & $3.349 E-5$ & $1.438 E-5$ \\
\hline & Error $\left(g_{0}\right)$ & $7.066 E-3$ & $1.397 E-4$ & $9.055 E-5$ & $7.367 E-4$ \\
\hline \multirow{4}{*}{$\begin{array}{l}\text { IMQ-RBFs } \\
(c=50)\end{array}$} & $\operatorname{RMS}(U)$ & $1.507 E-3$ & $5.384 E-4$ & $2.137 E-4$ & $1.560 E-4$ \\
\hline & Error $(U)$ & $3.546 E-3$ & $1.336 E-3$ & $5.354 E-4$ & $3.931 E-4$ \\
\hline & $\operatorname{RMS}\left(g_{0}\right)$ & $2.336 E-3$ & $8.666 E-4$ & $3.507 E-4$ & $2.562 E-4$ \\
\hline & Error $\left(g_{0}\right)$ & $5.902 E-3$ & $2.318 E-3$ & $9.491 E-4$ & $6.982 E-4$ \\
\hline \multirow{4}{*}{$\begin{array}{l}\text { MQ-RBFs } \\
(c=50)\end{array}$} & $\operatorname{RMS}(U)$ & $1.379 E-3$ & $5.107 E-4$ & $1.634 E-4$ & $1.343 E-4$ \\
\hline & Error $(U)$ & $3.243 E-3$ & $1.267 E-3$ & $4.094 E-4$ & $3.382 E-4$ \\
\hline & $\operatorname{RMS}\left(g_{0}\right)$ & $2.136 E-3$ & $8.221 E-4$ & $2.682 E-4$ & $2.220 E-4$ \\
\hline & Error $\left(g_{0}\right)$ & $5.398 E-3$ & $2.199 E-3$ & $7.257 E-4$ & $6.004 E-4$ \\
\hline \multirow{4}{*}{$\begin{array}{l}\text { IQ-RBFs } \\
(c=50)\end{array}$} & $\operatorname{RMS}(U)$ & $1.565 E-3$ & $3.116 E-4$ & $1.172 E-4$ & $1.342 E-5$ \\
\hline & Error $(U)$ & $3.683 E-3$ & $7.736 E-4$ & $2.951 E-4$ & $2.383 E-4$ \\
\hline & $\operatorname{RMS}\left(g_{0}\right)$ & $2.426 E-3$ & $5.013 E-4$ & $2.913 E-4$ & $2.205 E-4$ \\
\hline & Error $\left(g_{0}\right)$ & $6.130 E-3$ & $1.341 E-3$ & $6.371 E-4$ & $6.008 E-4$ \\
\hline
\end{tabular}

TABLE 9: Error and RMS errors for $U(x, y, t)$ on the intervals $x \in[0.1,1], y \in[0,1]$, and $t \in[0,1]$ for Example 2.

\begin{tabular}{|c|c|c|c|c|c|}
\hline Method & Error & $N=M=S=3$ & $N=6, M=4, S=5$ & $N=M=S=5$ & $N=5, M=6, S=5$ \\
\hline GA-RBFs & $\operatorname{RMS}(U)$ & $5.167 E-4$ & $5.678 E-5$ & $7.900 E-6$ & $5.923 E-6$ \\
\hline$(c=0.1)$ & Error $(U)$ & $7.048 E-4$ & $8.081 E-5$ & $1.125 E-5$ & $8.444 E-6$ \\
\hline IMQ-RBFs & $\operatorname{RMS}(U)$ & $9.580 E-5$ & $5.974 E-5$ & $5.022 E-6$ & $4.144 E-6$ \\
\hline$(c=50)$ & Error $(U)$ & $1.306 E-4$ & $8.502 E-5$ & $7.154 E-6$ & $5.909 E-6$ \\
\hline MQ-RBFs & $\operatorname{RMS}(U)$ & $4.735 E-5$ & $4.129 E-5$ & $1.013 E-5$ & $2.279 E-6$ \\
\hline$(c=50)$ & Error $(U)$ & $6.458 E-5$ & $7.015 E-5$ & $1.444 E-5$ & $3.250 E-6$ \\
\hline IQ-RBFs & $\operatorname{RMS}(U)$ & $1.291 E-4$ & $5.921 E-5$ & $3.043 E-6$ & $2.677 E-6$ \\
\hline$(c=50)$ & Error $(U)$ & $1.761 E-4$ & $8.427 E-5$ & $4.335 E-6$ & $4.243 E-6$ \\
\hline
\end{tabular}

Table 2 shows the comparison between the exact solution, RBFs solution and approximate solution result from method in [28] by Tikhonov regularization 0 th, 1 st, and $2 \mathrm{nd}$, and SVD with noiseless data. Table 3 shows this comparison with noisy data. Also, Table 4 shows the Error and RMS error values for $U(x, y, t)$ and $g_{0}(y, t)$ on the intervals $x \in[0,0.1]$, $y \in[0,1]$, and $t \in[0,1]$ for various values of $N, M$, and $S$. The corresponding results obtained for $U(x, y, t)$ on the intervals $x \in[0.1,1], y \in[0,1]$, and $t \in[0,1]$ are presented in Table 5. Figure 1 shows the plot of error for $U(x, y, 1)$ and $g_{0}(y, t)$ on the intervals $x \in[0,0.1], y \in[0,1]$, and $t \in[0,1]$. It can be obtained from Tables 4 and 5 and Figure 2 that the accuracy increases with the increase of the number of collocation points.

6.2. Example 2. As the second example, consider (1)-(2) with [28]: $x_{1}=0.1, t_{\text {fin }}=1, f(x, y)=\sin (x)+$ $\sin (y) g_{1}(y, t)=e^{-t}(\sin (1)+\sin (y)), h_{0}(x, t)=e^{-t} \sin (x)$, $h_{1}(y, t)=e^{-t}(\sin (x)+\sin (1))$, and $E(y, t)=e^{-t}(\sin (0.1)+$ $\sin (y))$ for which the exact solution is $u(x, y, t)=e^{-t}(\sin (x)+$ $\sin (y))$ and $g_{0}(y, t)=e^{-t} \sin (y)$.

Table 6 shows the comparison between the exact solution, RBFs solution and approximate solution result from method in [28] by Tikhonov regularization 0 th, 1st, and 2 nd, and SVD with noiseless data. Table 7 shows this comparison with noisy data. Furthermore, Table 8 shows the Error and RMS error values for $U(x, y, t)$ and $g_{0}(y, t)$ on the intervals $x \in$ $[0,0.1], y \in[0,1]$, and $t \in[0,1]$ for various values of $N$, $M$, and $S$. The corresponding results obtained for $U(x, y, t)$ on the intervals $x \in[0.1,1], y \in[0,1]$, and $t \in[0,1]$ are presented in Table 9. It can be obtained from Tables 8 and 9 that the accuracy increases with the increase of the number of collocation points. Also absolute error with different radial basis functions is depicted in Figure 2.

\section{Conclusion}

In the paper, an application of the RBFs for the solution of a two-dimension IHCP is presented. Problem consists in the calculation of temperature distribution in the domain, as well as in the reconstruction of functions describing the temperature on the boundary, when the temperature measurements in the domain are known. The present study successfully applies the numerical method to IHCPs and has been found stable with respect to small perturbation in the input data. The results of computing by radial basis function 
method are compared to that by the method in [28]. The results of numerical examples demonstrate that this method is more accurate than the method in [28].

\section{Conflict of Interests}

The authors declare that there is no conflict of interests regarding the publication of this paper.

\section{References}

[1] J. V. Beck, B. Blackwell, and S. R. Chair, Inverse Heat Conduction: Ill-Posed Problems, John Wiley \& Sons, New York, NY, USA, 1985.

[2] R. Siegel and J. R. Howell, Thermal Radiation Heat Transfer, Taylor \& Francis, New York, NY, USA, 4th edition, 1992.

[3] S. M. H. Sarvari, J. R. Howell, and S. H. Mansouri, "Inverse boundary design conduction-radiation problem in irregular two-dimensional domains," Numerical Heat Transfer B: Fundamentals, vol. 44, no. 3, pp. 209-224, 2003.

[4] O. M. Alifanov, Inverse Heat Transfer Problems, Springer, 1994.

[5] J. Baumeister, Stable Solution of Inverse Problems, Vieweg, 1987.

[6] H. W. Engl, M. Hanke, and A. Neubauer, Regularization of Inverse Problems, Kluwer Academic, Dordrecht, The Netherlands, 2000.

[7] A. Rieder, Keine Probleme mit Inversen Problemen, Vieweg, 2003.

[8] S. Chantasiriwan, "Comparison of three sequential function specification algorithms for the inverse heat conduction problem," International Communications in Heat and Mass Transfer, vol. 26, no. 1, pp. 115-124, 1999.

[9] D. Lesnic, L. Elliott, and D. B. Ingham, "Application of the boundary element method to inverse heat conduction problems," International Journal of Heat and Mass Transfer, vol. 39, no. 7, pp. 1503-1517, 1996.

[10] D. Lesnic and L. Elliott, "The decomposition approach to inverse heat conduction," Journal of Mathematical Analysis and Applications, vol. 232, no. 1, pp. 82-98, 1999.

[11] P. Jonas and A. K. Louis, "Approximate inverse for a onedimensional inverse heat conduction problem," Inverse Problems, vol. 16, no. 1, pp. 175-185, 2000.

[12] A. Frackowiak, N. D. Botkin, M. Ciałkowski, and K. Hoffmann, "A fitting algorithm for solving inverse problems of heat conduction," International Journal of Heat and Mass Transfer, vol. 53, no. 9-10, pp. 2123-2127, 2010.

[13] H. Molavi, A. Hakkaki-Fard, M. Molavi, R. K. Rahmani, A. Ayasoufi, and S. Noori, "Estimation of boundary conditions in the presence of unknown moving boundary caused by ablation," International Journal of Heat and Mass Transfer, vol. 54, no. 5-6, pp. 1030-1038, 2011.

[14] E. Hetmaniok, I. Nowak, D. Słota, and R. Wituła, "Application of the homotopy perturbation method for the solution of inverse heat conduction problem," International Communications in Heat and Mass Transfer, vol. 39, no. 1, pp. 30-35, 2012.

[15] S. Chantasiriwan, "An algorithm for solving multidimensional inverse heat conduction problem," International Journal of Heat and Mass Transfer, vol. 44, no. 20, pp. 3823-3832, 2001.

[16] N. Al-Khalidy, "A general space marching algorithm for the solution of two-dimensional boundary inverse heat conduction problems," Numerical Heat Transfer B, vol. 34, no. 3, pp. 339360, 1998.
[17] H.-J. Reinhardt, "A numerical method for the solution of twodimensional inverse heat conduction problems," International Journal for Numerical Methods in Engineering, vol. 32, no. 2, pp. 363-383, 1991.

[18] G. E. Bell, "An inverse solution for the steady temperature field within a solidified layer," International Journal of Heat and Mass Transfer, vol. 27, no. 12, pp. 2331-2337, 1984.

[19] C. Shu, H. Ding, and K. S. Yeo, "Solution of partial differential equations by a global radial basis function-based differential quadrature method," Engineering Analysis with Boundary Elements, vol. 28, no. 10, pp. 1217-1226, 2004.

[20] J. Li, "Application of radial basis meshless methods to direct and inverse biharmonic boundary value problems," Communications in Numerical Methods in Engineering with Biomedical Applications, vol. 21, no. 4, pp. 169-182, 2005.

[21] J. Li, "A radial basis meshless method for solving inverse boundary value problems," Communications in Numerical Methods in Engineering, vol. 20, no. 1, pp. 51-61, 2004.

[22] M. Dehghan and M. Tatari, "Determination of a control parameter in a one-dimensional parabolic equation using the method of radial basis functions," Mathematical and Computer Modelling, vol. 44, no. 11-12, pp. 1160-1168, 2006.

[23] K. Parand, S. Abbasbandy, S. Kazem, and J. A. Rad, "A novel application of radial basis functions for solving a model of firstorder integro-ordinary differential equation," Communications in Nonlinear Science and Numerical Simulation, vol. 16, no. 11, pp. 4250-4258, 2011.

[24] S. Kazem and J. A. Rad, "Radial basis functions method for solving of a non-local boundary value problem with Neumann's boundary conditions," Applied Mathematical Modelling, vol. 36, no. 6, pp. 2360-2369, 2012.

[25] S. U. Islam, S. Haqb, and A. Ali, "A meshfree method for the numerical solution of the RLW equation," Journal of Computational and Applied Mathematics, vol. 223, no. 2, pp. 997-1012, 2009.

[26] Y. C. Hon and T. Wei, "A meshless computational method for solving inverse heat conduction problem," International Series on Advances in Boundary Elements, vol. 13, pp. 135-144, 2002.

[27] M. Dehghan and A. Shokri, "A numerical method for solution of the two-dimensional sine-Gordon equation using the radial basis functions," Mathematics and Computers in Simulation, vol. 79, no. 3, pp. 700-715, 2008.

[28] R. Pourgholi, A. Esfahani, and M. Abtahi, "A numerical solution of a two-dimensional IHCP," Journal of Applied Mathematics and Computing, vol. 41, no. 1-2, pp. 61-79, 2013.

[29] J. R. Cannon, The One-Dimensional Heat Equation, AddisonWesley, Reading, Mass, USA, 1984.

[30] M. A. Golberg, C. S. Chen, and H. Bowman, "Some recent results and proposals for the use of radial basis functions in the BEM," Engineering Analysis with Boundary Elements, vol. 23, no. 4, pp. 285-296, 1999.

[31] S. Rippa, "An algorithm for selecting a good value for the parameter $c$ in radial basis function interpolation," Advances in Computational Mathematics, vol. 11, no. 2-3, pp. 193-210, 1999.

[32] A. H. D. Cheng, M. A. Golberg, E. J. Kansa, and Q. Zammito, "Exponential convergence and $\mathrm{H}-\mathrm{c}$ multiquadric collocation method for partial differential equations," Numerical Methods for Partial Differential Equations, vol. 19, pp. 571-594, 2003.

[33] G. E. Fasshauer and J. G. Zhang, "On choosing "optimal” shape parameters for RBF approximation," Numerical Algorithms, vol. 45, no. 1-4, pp. 345-368, 2007. 
[34] A. J. Khattak, S. I. A. Tirmizi, and S. U. Islam, "Application of meshfree collocation method to a class of nonlinear partial differential equations," Engineering Analysis with Boundary Elements, vol. 33, no. 5, pp. 661-667, 2009.

[35] M. Dehghan and A. Shokri, "A meshless method for numerical solution of the one-dimensional wave equation with an integral condition using radial basis functions," Numerical Algorithms, vol. 52, no. 3, pp. 461-477, 2009.

[36] E. Kreyszig, Introductory Functional Analysis with Applications, John Wiley \& Sons, New York, NY, USA, 1978.

[37] Z. M. Wu, "Radial basis functions in scattered data interpolation and the meshless method of numerical solution of PDEs," Journal of Engineering Mathematics, vol. 19, no. 2, pp. 1-12, 2002.

[38] Z. M. Wu and R. Schaback, "Local error estimates for radial basis function interpolation of scattered data," IMA Journal of Numerical Analysis, vol. 13, no. 1, pp. 13-27, 1993. 


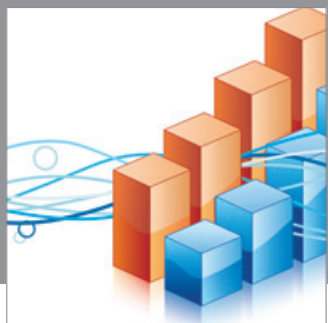

Advances in

Operations Research

mansans

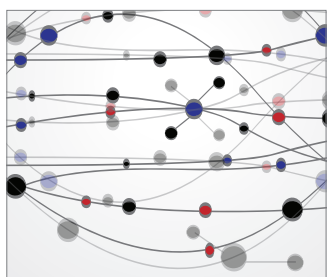

The Scientific World Journal
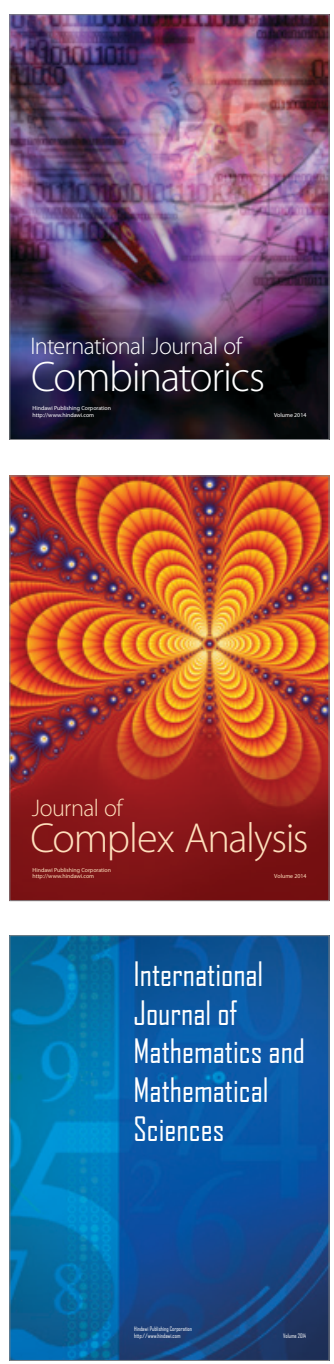
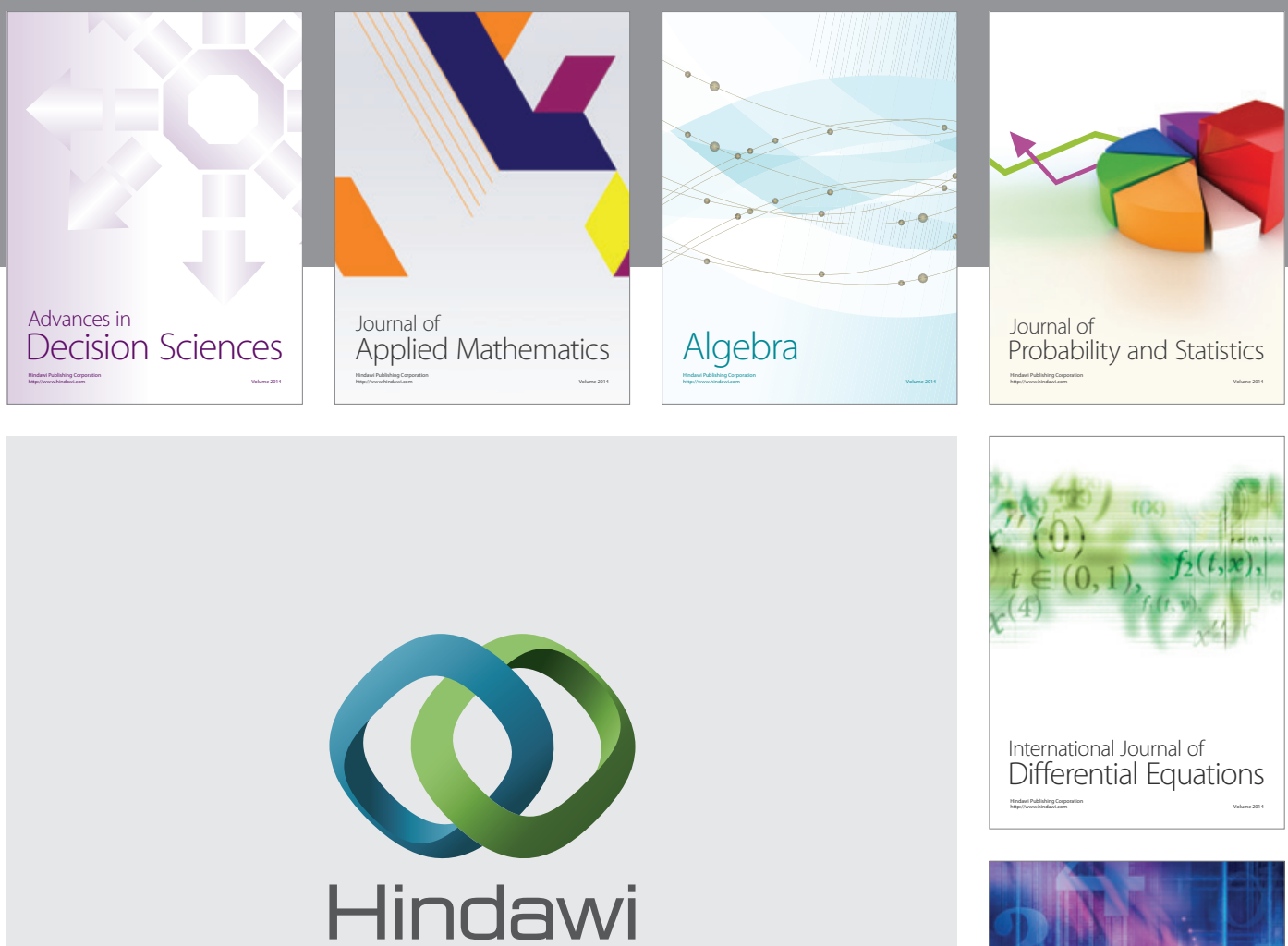

Submit your manuscripts at http://www.hindawi.com
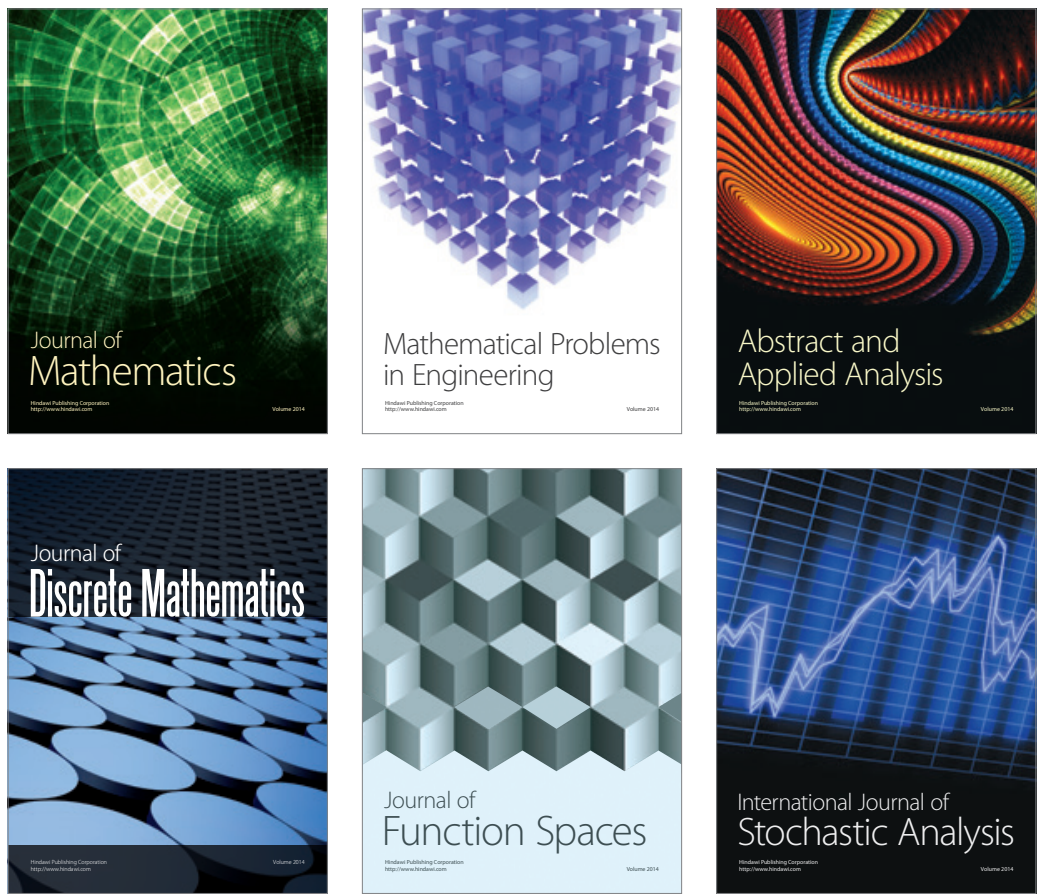

Journal of

Function Spaces

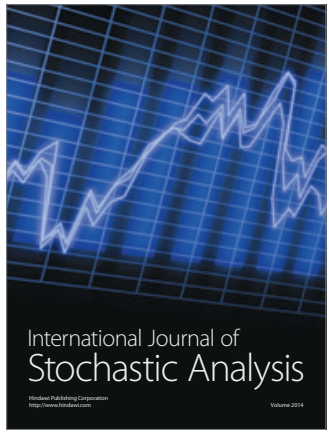

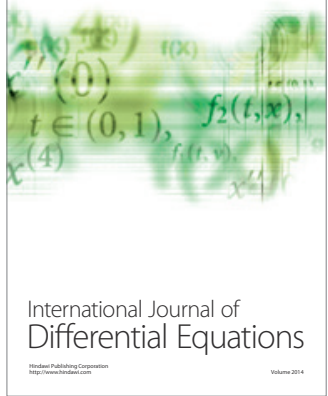
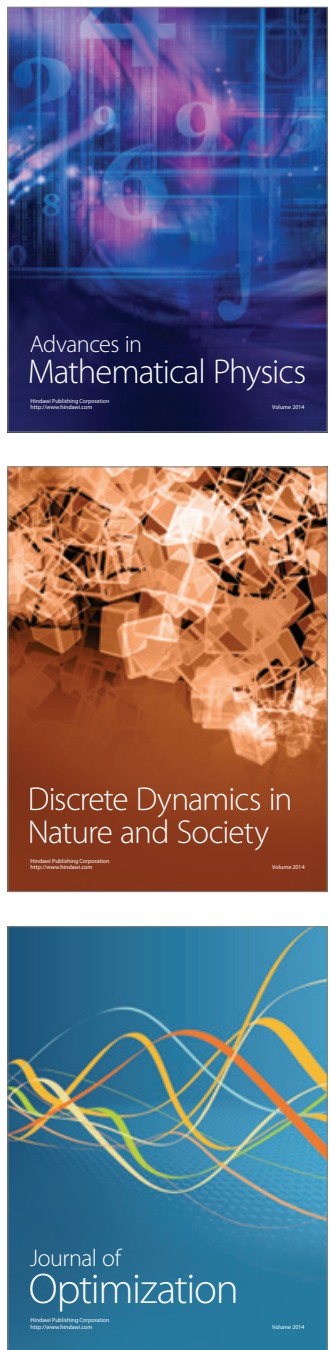